\title{
INFLUENCE OF DATA PROCESSING ON CYCLIC VARIATION OF INTEGRATED BACKSCATTER AND WALL THICKNESS IN STUNNED PORCINE MYOCARDIUM
}

\author{
Anton F. W. van der Steen, ${ }^{\dagger \ddagger}$ Hans Rijsterborgh, ${ }^{\dagger \ddagger}$ Charles T. Lancée ${ }^{\dagger}$ \\ Frits Mastik, ${ }^{\dagger}$ Rob Krams, ${ }^{\dagger}$ Pieter D. Verdouw ${ }^{\dagger}$ \\ Jos R. T. C. RoElANDT ${ }^{\dagger}$ and NICOLAAS BOM ${ }^{\dagger \dagger}$ \\ Department of Cardiology, Thoraxcenter, Erasmus University Rotterdam, Rotterdam, The Netherlands; and \\ Interuniversity Cardiology Institute of The Netherlands, Utrecht, The Netherlands
}

(Received 12 August 1996; in final form 18 November 1996)

\begin{abstract}
This study was performed to investigate the relationship between the cyclic variation of integrated backscatter and myocardial wall thickening in stunned myocardium. Different definitions of cyclic variation were evaluated to be able to compare with other studies. Ultrasound data were acquired from 10 openchested Yorkshire pigs $(25-33 \mathrm{~kg})$ at baseline, during regional ischemia and during $30 \mathrm{~min}$ of stunning, using a broadband ultrasound transducer (3-7 MHz) sutured directly upon the left ventricular myocardial wall. Cyclic variation of integrated backscatter and myocardial wall thickening were calculated using three definitions obtained from the literature. Independent of the definition, cyclic variation of wall thickness and integrated backscatter were blunted during acute ischemia and returned transiently to or above baseline during the first minute of reperfusion, followed by a gradual decrease to a level under baseline during stunning. An early return of the cyclic variation of the integrated backscatter was not observed in pigs, independent of the data processing used. The relationship between integrated backscatter and wall thickness was maintained. (c) 1997 World Federation for Ultrasound in Medicine \& Biology.
\end{abstract}

Key Words: Integrated backscatter, Cyclic variation, Wall thickening, Stunned myocardium, Data processing.

\section{INTRODUCTION}

The objective of this study was to obtain a better understanding of the phenomena concerned with stunning, variation in wall thickness and integrated backscatter, particularly the asynchronous recovery of cyclic variation of wall thickness and integrated backscatter.

The cyclic variation of ultrasound myocardial integrated backscatter during the cardiac cycle is widely regarded as a parameter to assess regional myocardial contractile performance (Barzilai et al. 1984; Glueck et al. 1985; Mottley et al. 1984; Sagar et al. 1987, 1988; Wear et al. 1986, 1989; Wickline et al. 1985a, $1985 \mathrm{~b}$ ). This cyclic variation of normal myocardial tissue as well as its disappearance during acute myocardial ischemia (Barzilai et al. 1984; Maderas et al. 1983; Miller et al. 1983; Mimbs et al. 1981) can be

Address correspondence to: Ton van der Steen, Ph.D., Laboratory of Experimental Echocardiography, Thorax Centre, Room Ee2302, P.O. Box 1738, 3000 DR Rotterdam, The Netherlands. Hans Rijsterborgh deceased 16 November 1993 explained by an empirical linear inverse relationship between myocardial integrated backscatter and myocardial wall thickness (Rijsterborgh et al. 1990, 1991; van der Steen et al. 1991). However, a simple relationship between backscatter level and wall thickness, regardless of the state of the myocardium, does not explain the normal cyclic variation of integrated backscatter observed in stunned myocardium, i.e., viable myocardial tissue, with depressed wall thickening during early reperfusion following acute ischemia (Milunski et al. 1989a, 1989b). Milunski et al. (1989a) performed in situ experiments on regionally stunned canine myocardium and found that the cyclic variation of integrated backscatter returned to normal values before the cyclic variation in wall thickness returned to baseline values. Furthermore, these investigators found in humans that the cyclic variation of integrated backscatter returned before a normal cyclic variation in wall thickness was observed (Milunski et al. 1989b). Hypothetically, this could be caused by the fact that, in stunned myocardium, perfusion recovers more rapidly 
than contractile function. However, other studies (Haasler et al. 1993; McPherson et al. 1986; Rijsterborgh et al. 1996) revealed that perfusion as such does not influence variation in integrated backscatter.

In this study, integrated backscatter and wall thickness as well as their cyclic variation were studied in open-chested Yorkshire pigs during baseline conditions, left anterior descending coronary artery (LADCA) occlusion and several stages of reperfusion. To be able to compare the results with those from studies performed by other groups, the cyclic variation was calculated using three different definitions as described and used by several authors (Mohr et al. 1989; Rijsterborgh et al. 1990; Sagar et al. 1987).

\section{METHODS}

\section{Animal preparation}

All experiments were performed in accordance with the Guiding Principles in the Care and Use of Animals as approved by the Council of the American Physiological Society (Office of Science and Health Reports No. (NIH) 80-23) and under the regulations of the Committee on Animal Experimentation of the Erasmus University Rotterdam.

After an overnight fast, 10 cross-bred Landrace $\times$ Yorkshire pigs (HVC, Hedel, The Netherlands) of either sex $(25-33 \mathrm{~kg})$ were sedated with $20 \mathrm{mg}$ $\mathrm{kg}^{-1}$ ketamine IM (AUV, Cuijk, The Netherlands), anaesthetized with $20 \mathrm{mg} \mathrm{kg}^{-1}$ sodium pentobarbital (Apharmo BV, Arnhem, The Netherlands), intubated and connected to a ventilator for intermittent positive pressure ventilation with a mixture of $\mathrm{O}_{2}$ and $\mathrm{N}_{2}(1: 2$, $\mathrm{vol} / \mathrm{vol})$. Respiratory rate and tidal volume were set and adjusted, when necessary, to keep arterial blood gases within the normal range (Soei et al. 1994). Catheters were placed in the superior vena cava for continuous infusion of $10-15 \mathrm{mg} \mathrm{kg}^{-1} \mathrm{~h}^{-1}$ sodium pentobarbital and in the descending aorta for withdrawal of blood samples and measurement of central aortic blood pressure. A 7-French Sensodyn micromanometertipped catheter (B. Braun Medical B.V., Uden, The Netherlands), inserted via the left carotid artery, was used to mcasure left ventricular pressure. After administration of $4 \mathrm{mg}$ of the muscle relaxant pancuronium bromidc (Organon Teknika B.V., Boxtel, The Netherlands) and a midline sternotomy, the left mammary vessels were ligated and the second left rib was removed for ease of further instrumentation, while the heart was suspended in a pericardial cradle. The ascending aorta was dissected free for placement of an electromagnetic flow probe (Skalar, Delft, The Netherlands ) for measurement of aortic blood flow. The proximal LADCA was dissected free for positioning of an atraumatic clamp. Rectal temperature was monitored throughout the experiment and maintained between $37^{\circ} \mathrm{C}$ and $38^{\circ} \mathrm{C}$ using external heating pads and appropriate coverage of the animals.

\section{Experimental protocol}

A broadband ultrasound transducer $(-20 \mathrm{~dB}$ bandwidth 3.2-7.2 MHz, Krautkramer-Branson, Lewistown, PA, USA) was sutured directly on the vascular free surface in the distribution area of the LADCA, while ensuring perpendicular insonification of the myocardial fibres. This set-up enabled the study of the same area throughout the entire experiment. However, it prevented spatial averaging.

After a stabilization period following completion of the surgical procedures, baseline recordings were made of pressures in the descending aorta (AP), left ventricle (LVP) and its first derivative ( $\mathrm{LVdP} / \mathrm{dt}$ ), ascending aortic blood flow (cardiac output, $\mathrm{CO}$ ), wall thickness and backscatter in the distribution area of the LADCA. The perfusion area of the LADCA was then stunned by $10 \mathrm{~min}$ of LADCA occlusion and $30 \mathrm{~min}$ of reperfusion. Due to technical timing difficulties, it was not possible to acquire the full series in all 10 pigs.

Measurements of wall thickness, backscatter properties and hemodynamics were subsequentially repeated at baseline $(n=10)$, after $10 \mathrm{~min}$ of occlusion $(n=9)$ and after $1(n=8), 3(n=7), 5(n=8)$, $10(n=10), 15(n=10), 20(n=10), 25(n=10)$ and $30 \mathrm{~min}(n=10)$ of reperfusion.

\section{Data acquisition}

The ultrasound transducer was connected to a custom-designed transmitter/receiver (input impedance $100 \Omega$, fixed gain $29 \mathrm{~dB},-6 \mathrm{~dB}$ cutoff frequencies 1.2 and $10 \mathrm{MHz}$ ). The amplified ultrasound signal was filtercd by a fifth order $10-\mathrm{MHz}$ low pass Chebyshev filter and connected to a dual channel digital oscilloscope (LeCroy 9310L). Ultrasound signals from the time interval of interest $(4.5-24.5 \mu \mathrm{s}$ following the transmitter pulse) were digitized with 8-bit resolution at a sample frequency of $25 \mathrm{MHz}$. In the other channel the left ventricular pressure was digitized. The digitized signals were stored in an IBM-compatible PC for off-line analysis.

Measurement sequences were generated by the PC. The heart rate was determined from the left ventricular pressure. The transmitter was triggered such that 51 traces could be acquired per heartbeat. A sequence of 10 heartbeats was acquired at each measurement. Heartbeats with cycle length outside the range of $90 \%-110 \%$ of the cycle length of the first beat of the measurements were omitted from further analysis. This occurred for $<1 \%$ of the beats. 


\section{Data processing}

Data were processed off line. Transmural myocardial wall thickness was calculated as the distance from the transducer on the epicardial to the endocardial wall. To locate the endocardial wall, the recorded ultrasound signals were displayed in M-mode and the wall was traced manually in every cardiac cycle by an observer using the computer system's mouse. The timing scale of all measurements was rescaled (based on the pressure curve) along the cardiac cycle from the beginning of systole $(0 \%)$ to the end of diastole ( $100 \%)$, with increments of $2 \%$ of the cardiac cycle.

Ultrasound spectra were extracted from the signals after applying a split cosine bell window ( $p=$ 0.1 ) of 5- $\mu$ s length (Bloomfield 1976), excluding the endocardial wall. Integrated backscatter was calculated by averaging the spectra in the frequency range from $3.2-7.2 \mathrm{MHz}$ (i.e., the $-20 \mathrm{~dB}$ bandwidth of the transducer).

Mean values and standard deviations of the integrated backscatter, wall thickness and left ventricular pressure were calculated for every $2 \%$ increment of the cardiac cycle.

Since various definitions of cyclic variation of integrated backscatter appeared in the literature, the cyclic variations of integrated backscatter and wall thickness were calculated three different ways (Fig. 1). Because of the empirical inverse relationship between wall thickness and integrated backscatter (Maderas et al. 1983; Miller et al. 1983; Mimbs et al. 1981; Rijsterborgh et al. 1990, 1991; van der Stcen ct al. 1991 ), all models were inverted before calculating cyclic variation in wall thickness in all three methods.

\section{End-systolic and end-diastolic differences (ESED)}

This method was described by Rijsterborgh et al. (1990). End systole was defincd as the time interval from $30 \%-46 \%$ cardiac cycle and end diastole as being from $80 \%-96 \%$, each interval containing nine samples. End-systolic and end-diastolic values of integrated backscatter and wall thickness were calculated by averaging the mean values of the parameters over these intervals. Cyclic variation was defined as the difference between the values in those intervals (Fig. 1 , panels $a$ and $b$ ).

\section{Selective power spectrum comparison (SPSC)}

This method describes the cyclic variation of integrated backscatter as a smoothed square wave function and determines its amplitude from the harmonics of the spectrum from this square wave function. It was described in detail by Mohr et al. (1989). In summary, it is described as follows.

(a) First the average length of systole and the full cardiac cycle were determined. [Mohr et al. (1989) defined these as the average time interval from the beginning of each QRS complex to the end of each $T$ wave and the average period between two R tops. We extracted them from the left ventricular pressure. This is the only minor adaption to the method as it was introduced.]

(b) Then an objective model function was fitted to the data. This was a square wave function with a high value during the systolic interval and low value during the rest of cardiac cycle. The magnitude of the cyclic variation of the model is initially arbitrary. Both data and model were filtered by a binomial coefficient digital filter. The smoothed square wave was offset to have zero mean over the cardiac period. The data were truncated at both ends to retain an integral number of heartbeats. Offset and sloping trends in the data were removed by applying a least square fit to the filtered data and subtracting this fit from the data.

(c) An eventual delay between the model and the data was removed by cross-correlating them and shifting the model over the time interval at which the maximum cross-correlation occurred.

Mohr et al. (1989) normalized this time delay to the systolic length and added 0.5 to this value for display purposes. In this way, the delay is displayed as the delay between the start of the systolic interval and the nadir of cyclic variation.

(d) Then both model and data were Fourier transformed. Since the model was a periodical smoothed square wave containing an integral number of heartbeats, the spectrum of the model carries power over the fundamental frequency (which corresponds to the frequency of the heartbeat) and its higher harmonics.

Finally, the amplitude of the model has to be determined. This was done in two steps (e and $f$ ).

(e) At each harmonic, the power in the data was compared to the power in the model. There were two criteria for excluding a harmonic: (1) the power of the particular model harmonic was $<1 \%$ of the power at the maximal model harmonic; and (2) the power at the particular harmonic in the data was greater than the power in the fundamental of the data. Typically, 3-6 harmonics remained for further analysis. The power in each of these harmonics of the data was divided by the power in the corresponding harmonic in the model. These power ratios were weighted by the corresponding power content of the data and then averaged.

(f) Finally, the cyclic variation is defined as the square root of this weighted average multiplied by 
relative
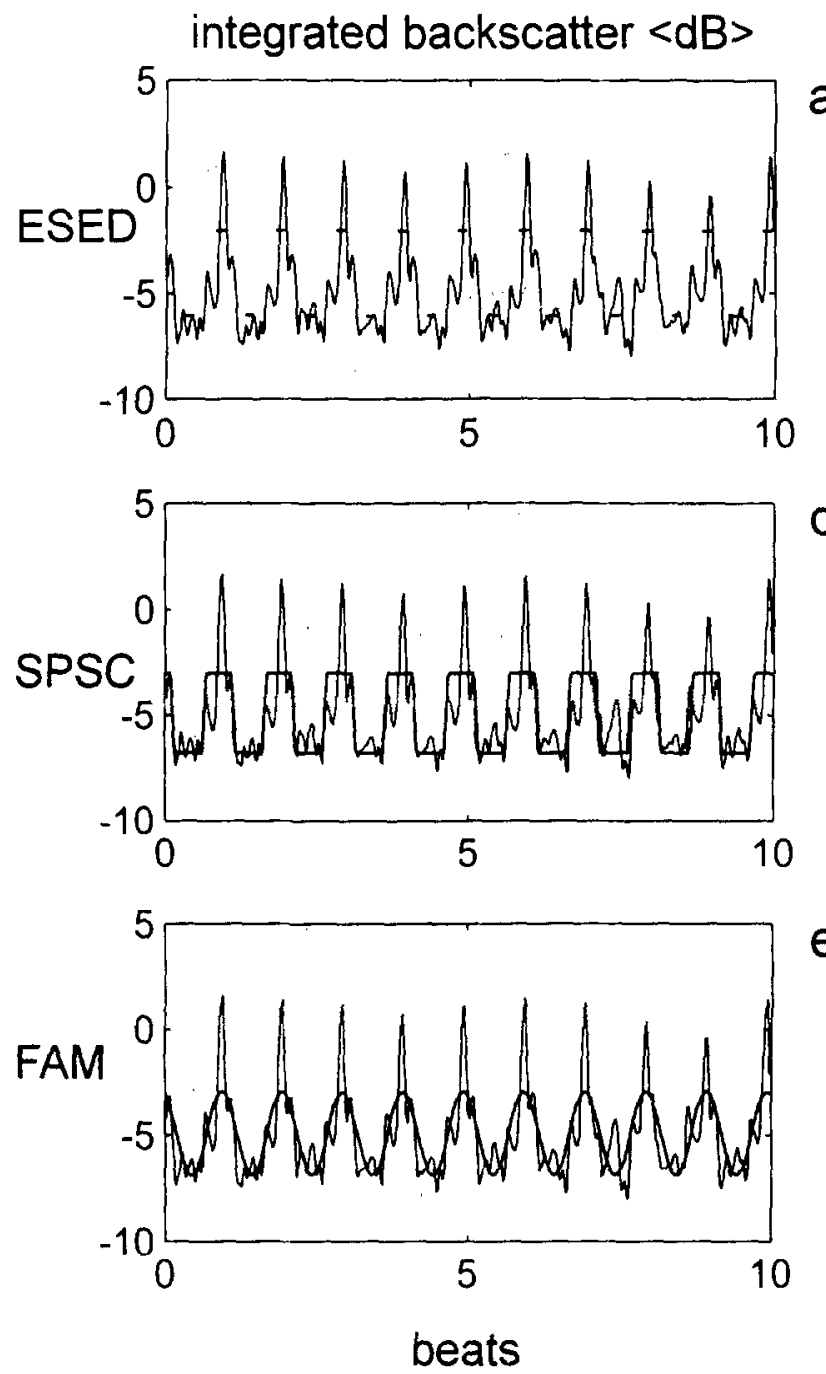

a

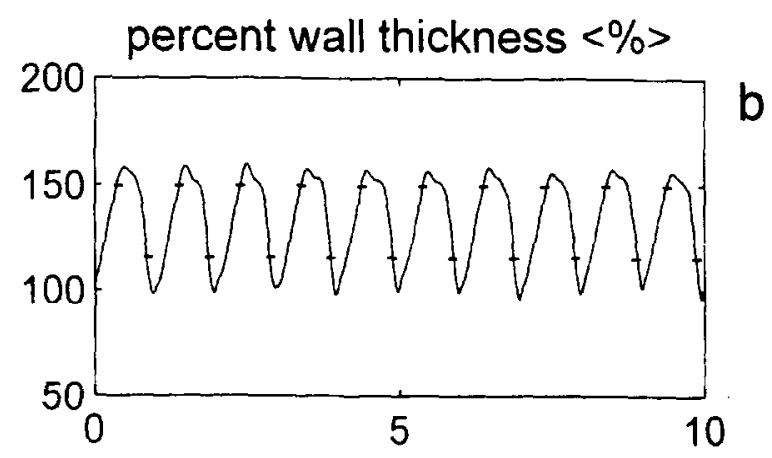

C

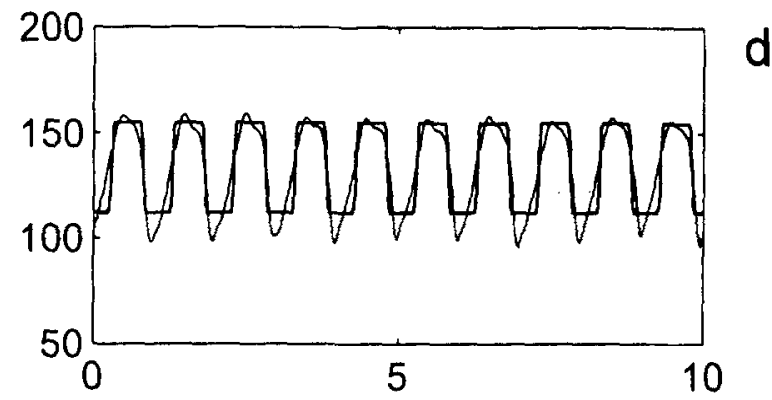

e

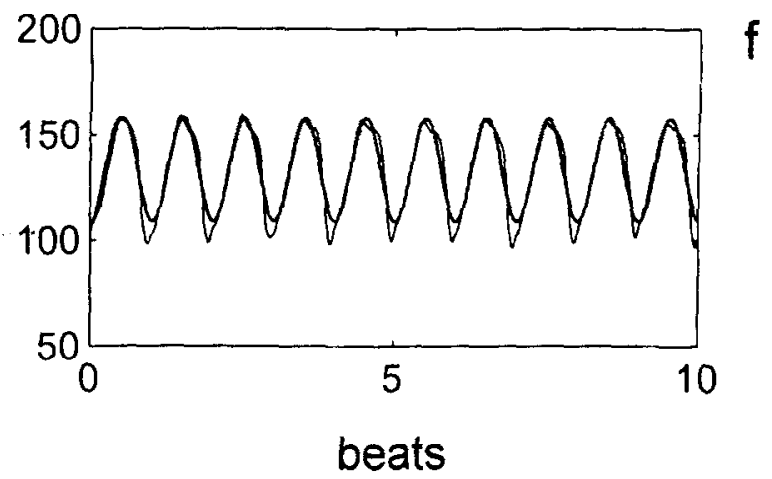

Fig. 1. The various methods of calculating cyclic variation of integrated backscatter and wall thickness in normally perfused myocardium. Data are from a sequence of 10 heartbeats obtained from a representative pig. The values for the cyclic variation are: (a) end-systolic end-diastolic (ESED) integrated backscatter: $4.00 \mathrm{~dB}$; (b) ESED wall thickness: $34.0 \%$; (c) Selective power spectrum comparison (SPSC) integrated backscatter: $3.78 \mathrm{~dB}$; (d) SPSC wall thickness: $42.8 \%$; (e) Fourier component of amplitude modulation (FAM) integrated backscatter: 3.95 $\mathrm{dB}$; (f) FAM wall thickness: $48.9 \%$.

the arbitrarily chosen magnitude of the model (Fig. 1, panels $\mathrm{c}$ and d).

\section{Fourier component of amplitude modulation (FAM)}

This method was introduced by Sagar et al. (1987). A sine wave whose period matched the cardiac cycle was fitted to the data. First, the delay of the sine wave relative to the cardiac cycle was established by a crosscorrelation technique. This delay was also normalized to the systolic length and 0.5 was added. Secondly, the amplitude of the sine wave was estimated. Cyclic variation was defined as twice the amplitude of this sine wave (Fig. 1, panels e and f).
FAM will give the same results as SPSC when only the fundamental is taken into account, using the SPSC method.

\section{Statistics}

Cyclic variation of integrated backscatter and wall thickness as well as delay of maximal wall thickening and integrated backscatter were calculated using the three methods described above at baseline, after 10 min of occlusion and after the aforementioned time points of reperfusion. At each point, the averages and standard deviations were calculated for 10 pigs. All parameters were tested for normality using a Wilk- 
Table 1a. Cyclic variation of wall thickness according to the three methods during the different phases of the experiments.

\begin{tabular}{llll}
\hline & ESED & \multicolumn{1}{c}{ FAM } & \multicolumn{1}{c}{ SPSC } \\
\hline Baseline & $37.9(11.2)$ & $36.6(8.6)$ & $32.5(8.0)$ \\
10 min occlusion & $-2.2(2.8)^{*}$ & $18.4(7.4)^{*}$ & $22.7(13.6)$ \\
1 min reperfusion & $37.8(7.2)$ & $36.1(5.5)$ & $33.8(6.8)$ \\
3 min reperfusion & $26.4(11.5)$ & $32.8(6.7)$ & $30.6(7.1)$ \\
5 min reperfusion & $21.8(10.5)^{*}$ & $31.0(4.9)$ & $28.4(5.2)$ \\
10 min reperfusion & $23.5(9.2)^{*}$ & $32.2(4.0)$ & $30.0(5.2)$ \\
15 min reperfusion & $23.7(10.1)^{*}$ & $31.6(4.3)$ & $29.3(4.7)$ \\
20 min reperfusion & $24.4(9.5)^{*}$ & $31.3(4.4)$ & $28.5(4.4)$ \\
25 min reperfusion & $24.0(9.2)^{*}$ & $30.5(4.9)$ & $27.8(4.8)$ \\
30 min reperfusion & $23.9(9.2)^{*}$ & $31.5(3.8)$ & $29.0(4.3)$ \\
\hline
\end{tabular}

Values are mean $(\mathrm{SD})\langle \%\rangle$.

$*$ Values that differ significantly from baseline $(p<0.05)$.

Table 1b. Cyclic variation of integrated backscatter according to the three methods during the different phases of the experiments.

\begin{tabular}{llll}
\hline & ESED & FAM & SPSC \\
\hline Baseline & $3.3(3.4)$ & $4.1(2.7)$ & $4.2(2.2)$ \\
10 min occlusion & $0.3(0.8)^{*}$ & $1.9(1.2)^{*}$ & $3.1(2.2)$ \\
1 min reperfusion & $4.1(2.3)$ & $4.2(1.6)$ & $4.5(2.0)$ \\
3 min reperfusion & $2.6(1.8)$ & $4.9(1.8)$ & $6.0(1.9)$ \\
5 min reperfusion & $1.4(1.6)$ & $4.6(2.2)$ & $4.8(2.0)$ \\
10 min reperfusion & $2.0(1.6)$ & $4.1(2.5)$ & $4.6(1.9)$ \\
15 min reperfusion & $1.8(1.8)$ & $4.4(2.6)$ & $4.8(2.5)$ \\
20 min reperfusion & $2.0(1.9)$ & $4.4(2.6)$ & $4.7(2.5)$ \\
25 min reperfusion & $2.2(1.8)$ & $4.2(2.4)$ & $4.6(2.3)$ \\
30 min reperfusion & $2.2(1.6)$ & $4.3(2.6)$ & $4.4(2.0)$ \\
\hline
\end{tabular}

Values are mean (SD) $\langle\mathrm{dB}\rangle$.

$*$ Values that differ significantly from baseline $(p<0.05)$.

Shapiro test at each reperfusion time and during the whole experiment. Significant difference from baseline was calculated using paired $t$-tests $(p<0.05)$.

\section{RESULTS}

The Wilk-Shapiro test revealed that the observations per reperfusion time were normally distributed, but because of the experimental design, the parameter set over the full experiment was not normally distributed. The histogram will be trimodally distributed ( see Figs. 2-6). One peak will appear around the values that occur after $10 \mathrm{~min}$ of occlusion, one peak around the values shortly after initial reperfusion (1 min) and the largest peak around the remaining values.

Numerical values as well as significant differences from baseline values $(p<0.05)$ of cyclic variation in wall thickness and integrated backscatter are listed in Tables $1 \mathrm{a}$ and $1 \mathrm{~b}$ for all three methods.

Figure 2 shows the cyclic variation of wall thickness (upper panel) and integrated hackscatter (lower panel) during the episodes of the experiments calculated as the difference between end-systolic and enddiastolic values (ESED). This is the classical way of calculating the cyclic variation in wall thickness. The pattern displayed in Fig. 2 (top) shows all the hallmarks of stunning (Brand et al. 1992; Hearse 1995): a loss of wall thickening during occlusion, followed by a prompt but transient recovery immediately upon reperfusion, followed by a sustained period of impaired wall thickening.

Figure 3 shows the cyclic variation of wall thickness (upper panel) and integrated backscatter (lower panel) during the episodes of the experiments calculated using the selective power spectrum comparison (SPSC).

Figure 4 shows the cyclic variation of wall thickness (upper panel) and integrated backscatter (lower panel) during the episodes of the experiments assessed by calculating the Fourier component of amplitude modulation (FAM).

Table 1c. Delay of maximal cyclic variation of wall thickness according to FAM and SPSC during the different phases of the experiments.

\begin{tabular}{lllll}
\hline & FAM WT & FAM IB & SPSC WT & SPSC IB \\
\hline Baseline & $0.98(0.10)$ & $1.08(0.21)$ & $0.97(0.10)$ & $1.08(0.20)$ \\
10 min occlusion & $1.37(0.31)^{*}$ & $1.26(0.41)$ & $1.42(0.15)^{*}$ & $1.29(0.27)$ \\
1 min reperfusion & $1.01(0.08)$ & $1.04(0.09)$ & $1.02(0.07)$ & $1.08(0.07)$ \\
3 min reperfusion & $1.11(0.15)^{*}$ & $1.19(0.18)$ & $1.11(0.12)^{*}$ & $1.16(0.16)$ \\
5 min reperfusion & $1.19(0.10)^{*}$ & $1.28(0.09)$ & $1.17(0.12)^{*}$ & $1.26(0.15)$ \\
10 min reperfusion & $1.15(0.10)^{*}$ & $1.10(0.35)$ & $1.13(0.13)^{*}$ & $1.15(0.27)$ \\
15 min reperfusion & $1.15(0.09)^{*}$ & $1.22(0.13)$ & $1.13(0.12)^{*}$ & $1.23(0.17)^{*}$ \\
20 min reperfusion & $1.14(0.08)^{*}$ & $1.22(0.09)$ & $1.11(0.10)^{*}$ & $1.20(0.14)^{*}$ \\
25 min reperfusion & $1.13(0.09)^{*}$ & $1.19(0.11)$ & $1.11(0.11)^{*}$ & $1.18(0.18)$ \\
30 min reperfusion & $1.14(0.10)^{*}$ & $1.16(0.14)$ & $1.11(0.11)^{*}$ & $1.20(0.17)$ \\
\hline
\end{tabular}

Values are mean (SD).

* Values that differ significantly from baseline $(p<0.05)$

$\mathrm{IB}=$ integrated backscatter; $\mathrm{WT}=$ wall thickness. 

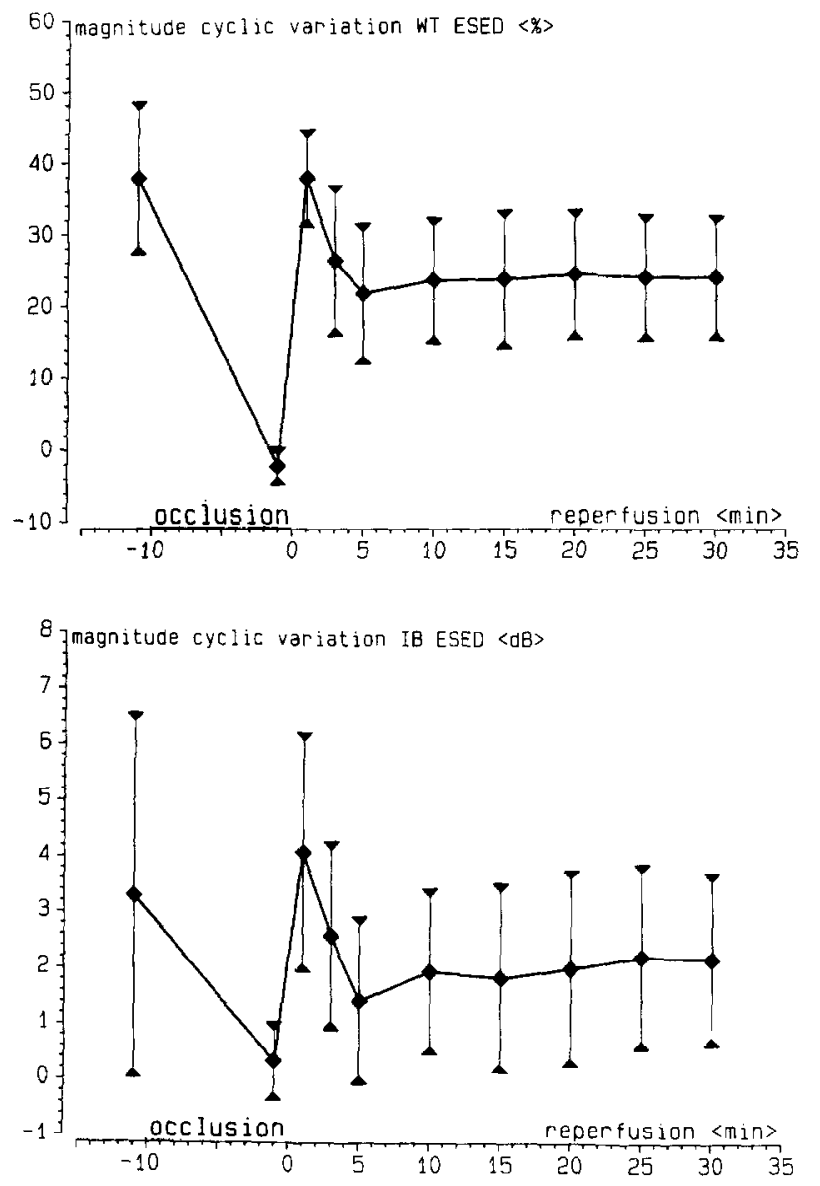

Fig. 2. The cyclic variation of wall thickness (upper panel) and integrated backscatter (lower panel) during the episodes of the experiments calculated as the end-systolic and enddiastolic difference (ESED).

All three methods reveal the same trend: the cyclic variations in wall thickness and integrated backscatter were blunted after $10 \mathrm{~min}$ of occlusion. After a short period of reperfusion, an overshoot in cyclic variation occurred and, after a longer period, it returned to a value that does not change between 5 and $30 \mathrm{~min}$. ESED reveals a relatively lower cyclic variation in wall thickness and integrated backscatter during stunning, as compared to the other two methods of calculating the cyclic variation. For ESED, the cyclic variation in wall thickness is significantly lower during stunning as compared to baseline, while the difference in cyclic variation in integrated backscatter is not significant. This is due to the lower relative standard deviation of the cyclic variation in wall thickness.

Return of cyclic variation in wall thickness and integrated backscatter occurred simultaneously, independent of the method used.

Figures 5 and 6 show the time delay, normalized to the systolic interval, of wall thickening and inte- grated backscatter relative to the beginning of systole as calculated from SPSC and FAM, respectively. The numerical values as well as significant differences from baseline values $(p<0.05)$ are listed in Table 1c.

\section{DISCUSSION}

Many studies have described phenomena that are related or potentially related to myocardial ultrasonic integrated backscatter and its variation during the cardiac cycle (Barzilai et al. 1984; Glueck et al. 1985; Hoffmeister et al. 1991; Maderas et al. 1988; Mottley and Miller 1988; Mottley et al. 1984; Recchia et al. 1993, 1995; Sagar et al. 1987, 1988, 1990; Wear et al. 1986, 1989; Wickline et al. 1985a, 1985b, 1991, 1992). An important observation has been the empirical inverse linear relation between wall thickness and integrated backscatter (Rijsterborgh et al. 1990, 1991; van der Steen et al. 1991). This relation does not seem to hold in all cases (Milunski et al. 1989a, 1989b).
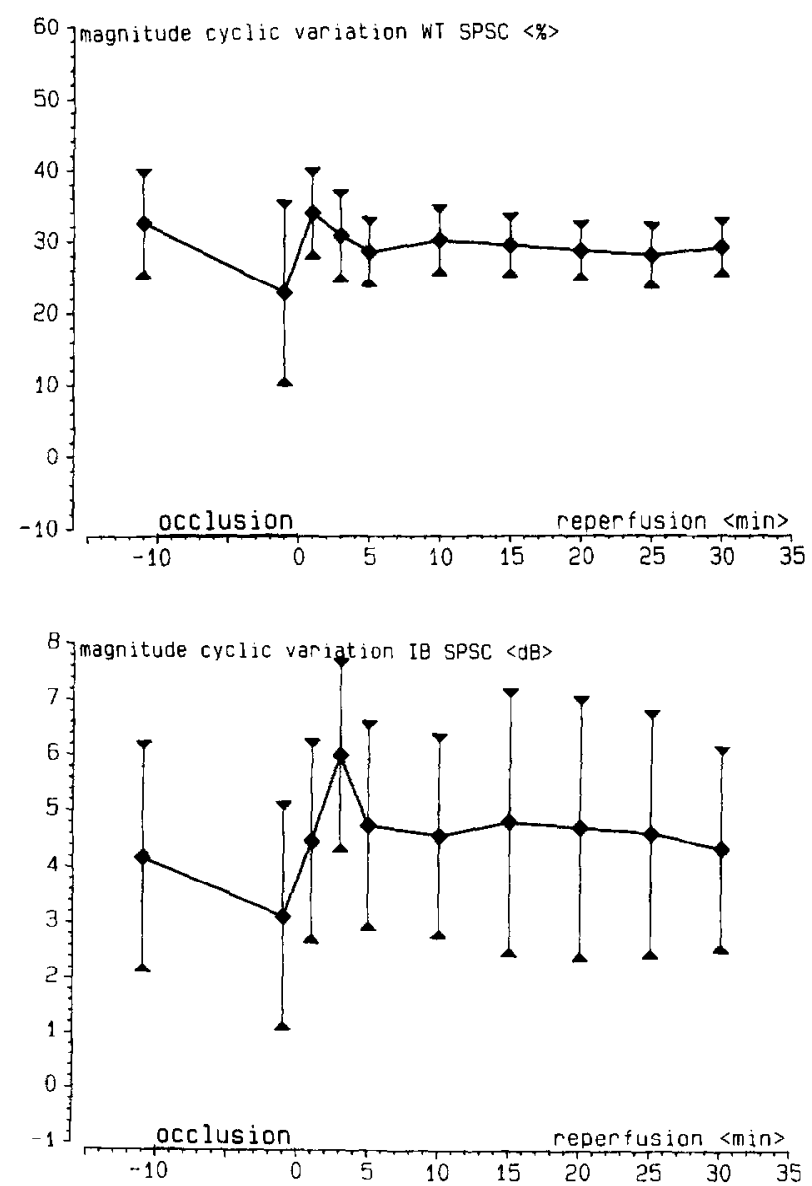

Fig. 3. The cyclic variation of wall thickness ( upper panel) and integrated backscatter (lower panel) during the episodes of the experiments calculated using the selective power spectrum comparison (SPSC). 

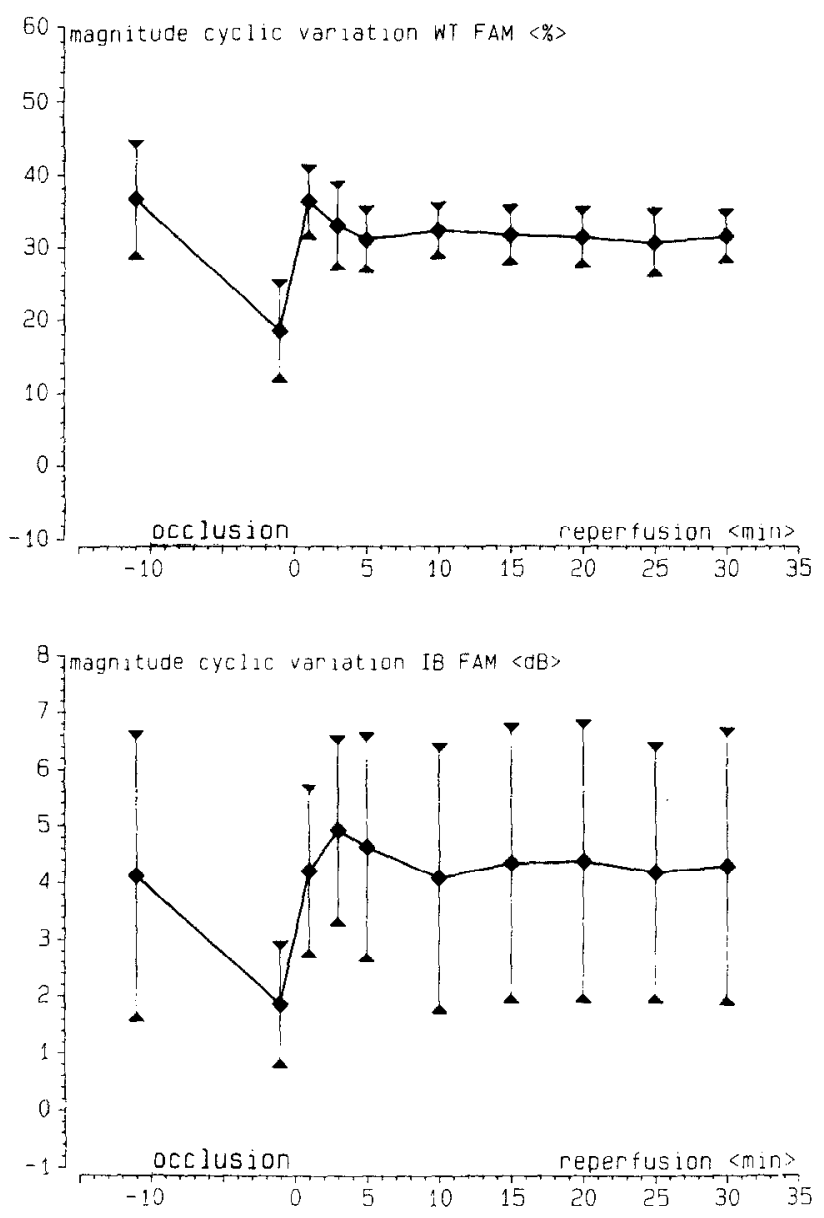

Fig. 4. The cyclic variation of wall thickness (upper panel) and integrated backscatter (lower panel) during the episodes of the experiments assessed by calculating the Fourier component of amplitude modulation (FAM).

One specific situation where integrated backscatter may have additional value over wall thickness measurements is to detect stunned but viable myocardium. Milunski et al. (1989a) found after coronary occlusion for $15 \mathrm{~min}$ an earlier return of cyclic variation of integrated backscatter compared to the variation in wall thickness in mongrel dogs. In patients, Milunski et al. (1989b) noticed a variation in integrated backscatter where a variation in wall thickness could not yet be detected. In earlier studies from our laboratory, an empirical relationship between wall thickness and integrated backscatter was measured during a variety of conditions of pig myocardium (van der Steen and Rijsterborgh 1996), including normal myocardium (Lancée et al. 1988; Rijsterborgh et al. 1993), fresh isolated myocardium ( van der Steen et al. 1991), early ischemic myocardium (Rijsterborgh et al. 1990, 1991 ), isolated heart myocardium under isovolumetric contraction (Rijsterborgh et al. 1996) and stunned myocardium (van der Steen et al. 1995). Our studies and the results of Milunski et al. (1989b) seem to contradict. We therefore repeated a study in pigs in which myocardial stunning was produced, and we analyzed the results using three standard definitions of cyclic variation of wall thickness and integrated backscatter.

\section{Comparison of different definitions}

In this study, it is shown that results obtained by these three definitions are closely related. There are a few principal differences. The definition as used by Rijsterborgh et al. (1990) (ESED) is the most straightforward one. It does not, however, include the influence of an eventual time shift between the rise of integrated backscatter or wall thickness under different circumstances. Another disadvantage is that it uses only a limited part of the data. The method as introduced by Mohr et al. (1989) (SPSC) includes the time shift and uses all data. However, it presumes that the
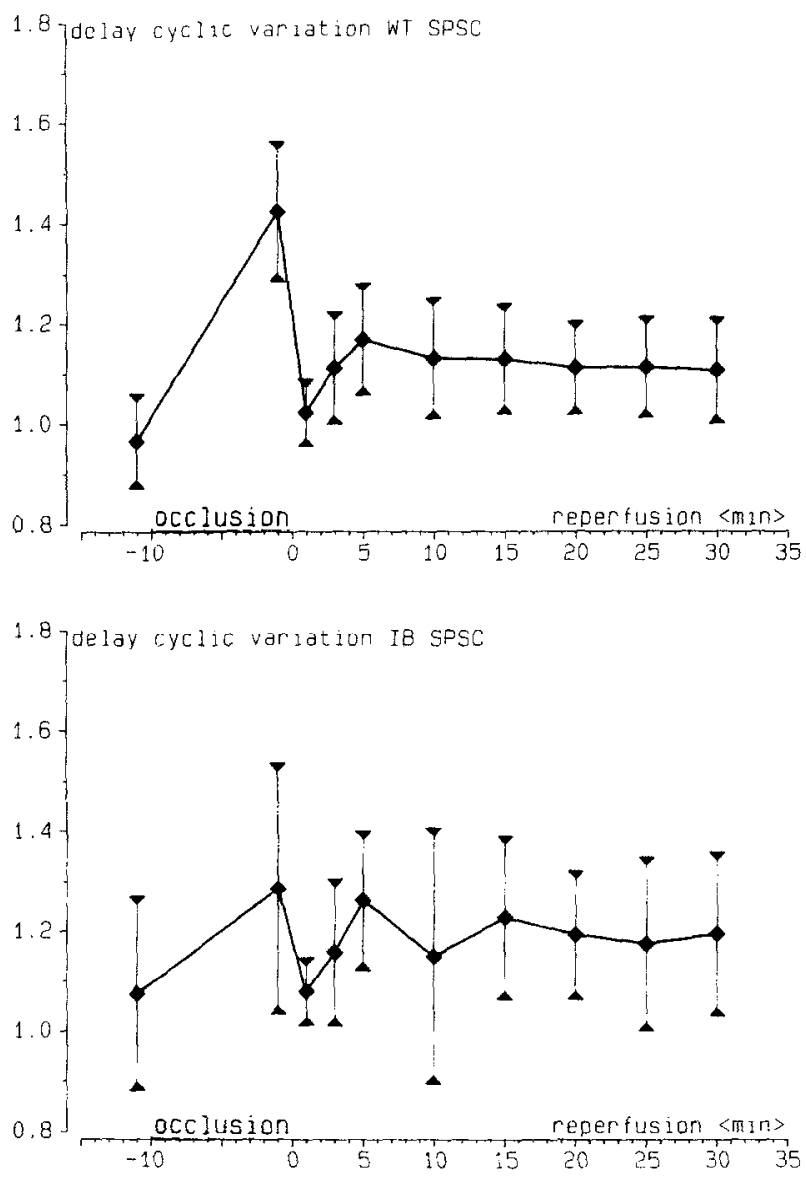

Fig. 5. The time delay of wall thickening and integrated backscatter normalized to the systolic interval and shifted half a systolic interval from the onset of contraction as calculated from the selective power spectrum comparison (SPSC). 

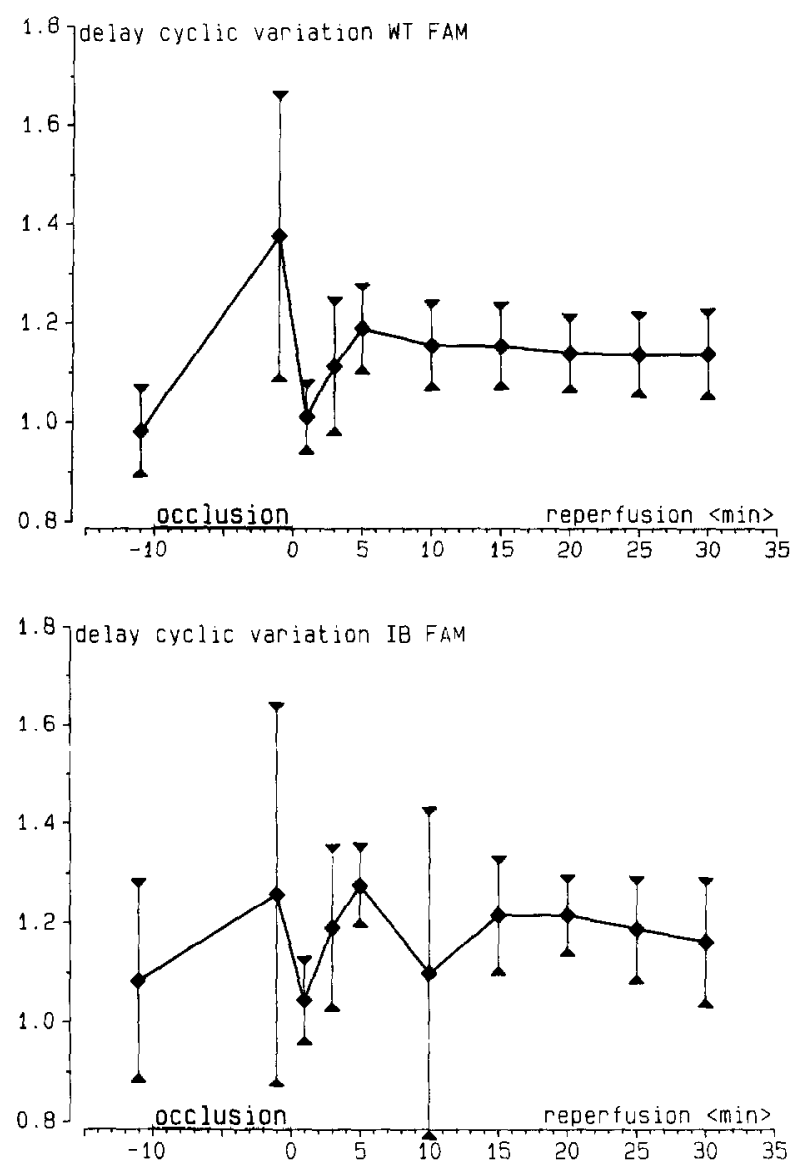

Fig. 6. The time delay of wall thickening and integrated backscatter normalized to the systolic interval and shifted half a systolic interval from the onset of contraction as calculated from the Fourier component of amplitude modulation (FAM).

data can be described by a smoothed square wave, an assumption to which we could not always subscribe. Furthermore, there are two criteria for omitting a harmonic from the analysis that are both critically present in the analysis: (1) the power in a harmonic should be more than $1 \%$ of the power at the maximal model harmonic; and (2) the power of a harmonic should not excecd the power of the fundamental harmonic. The last criterion especially occurs often in ischemic myocardium. The method of Sagar et al. (1990) (FAM) presumes that the signals could be described by a sine wave. This means that it excludes the influence of the second harmonic in the first place, which is often predominant in ischemic myocardium.

Including a time delay in wall thickness and integrated backscatter gives a more complete description of the phenomena, but it includes a few delicate side effects. This is illustrated by Fig. 7, where it can be seen that, during coronary occlusion, cyclic variations in both integrated backscatter and wall thickness are

Volume 23, Number 3, 1997

blunted (data without solid line). Furthermore, it can be seen that, in mid-diastole $(70 \%-80 \%)$, an increase in wall thickness appears. This paradoxical wall motion is not closely related to the contractility of the region of myocardium under study. As can be seen in Table 1c, the delay in wall thickening, and thus the magnitude, is determined by this paradoxical wall motion. This large shift in delay in case of occlusion and a smaller one in case of stunning was also reported by Bolli et al. (1988). Another problem is the dissociation in delay of wall thickening and integrated backscatter, which can be seen in Table 1c. This means that the integrated backscatter and wall thickening may be taken from different parts of the cardiac cycle.

Although the three analysis methods are not iden-
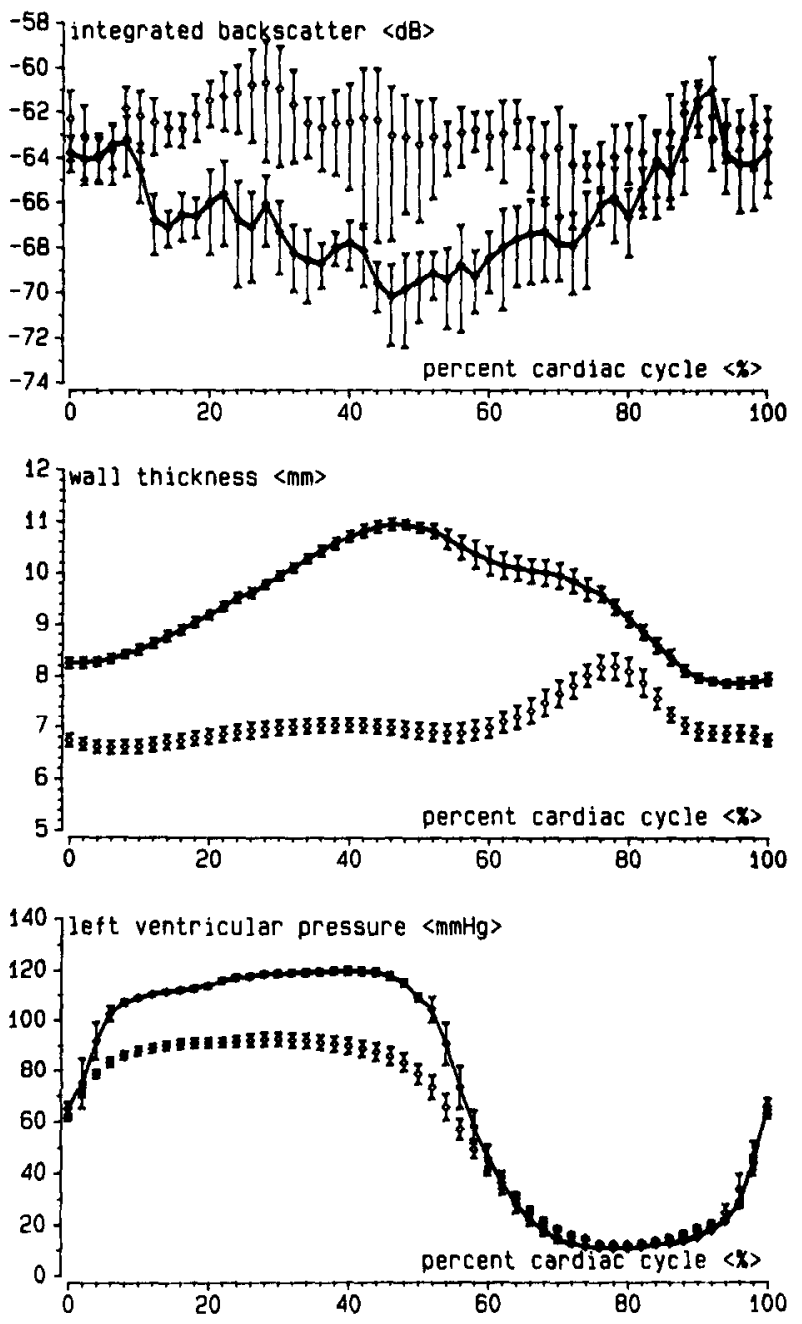

Fig. 7. Relative integrated backscatter, percent wall thickening and left ventricular pressure as a function of cardiac cycle. Shown are mean values (diamonds) and standard deviations (bars) of 10 cardiac cycles of a pig during baseline (connected with a solid line) and after $10 \mathrm{~min}$ of occlusion. [Reproduced with permission from Rijsterborgh et al. (1991).] 
tical, they reveal basically the same result. There are some differences: using ESED, the cyclic variation in integrated backscatter at baseline is somewhat lower than that described by the other two methods. Furthermore, it remains lower after reperfusion. This could be explained by the inclusion of the time delay in the latter two methods. In Figs. 5 and 6, it can be seen that coronary occlusion introduces an extra time delay in maximum integrated backscatter during the cardiac cycle, which does not fully return during the period of reperfusion under study. This means that maximal and minimal integrated backscatter do not occur at end diastole and end systole, which blunts the cyclic variation according to ESED. The same effect could be the cause for the observation that the maximal cyclic variation is found after $1 \mathrm{~min}$ according to ESED but after $3 \mathrm{~min}$ for SPSC and FAM. Figures 5 and 6 show that the delay varies most in this time interval, so it is not surprising that a method that does not include this delay reveals somewhat different results. As a result of these differences, a dissociation of cyclic variation of integrated backscatter and wall thickness will occur if they are not determined by the same method.

An early return of the cyclic variation of integraled backscatter in stunned myocardium could not be observed in Yorkshire pigs, independent of the method of data processing. The present results are in accordance with our earlier findings and indicate that the relationship between integrated backscatter and wall thickness is maintained, even during stunning. The discrepancy with the results by Milunski et al. (1989a) could possibly be explained by the choice of the animal. The coronary bed of the heart of dogs is characterized by the presence of collaterals that are absent in pigs. This results in a larger decrease of myocardial wall thickening in the pig as compared to the dog for similar occlusion periods. To facilitate comparison between our study and that of Milunski et al. (1989a), we occluded for $10 \mathrm{~min}$, while they occluded for $15 \mathrm{~min}$. However, the resulting cardiac dysfunction in our study was far less than noted in their study, implying that the differences might be related to the degree of stunning. Another explanation is the fact that they used SPSC for calculation of the cyclic variation in integrated backscatter while they used ESED for the cyclic variation in wall thickness. As stated above, this may cause a dissociation.

O'Brien et al. (1995) performed in situ measurements on mongrel dogs in which they compared absolute values of integrated backscatter at $5 \mathrm{MHz}$ in normal, stunned and ischemic myocardium. They occluded the LAD for 15 min to provoke stunning, in accordance with the measurements of Milunski et al (1989a). They measured absolute backscatter levels after 180 min of reperfusion and did not find a difference in stunned and normal myocardium, which is in accordance with both our measurements and those of Milunski et al. (1989a).

In conclusion, we did not find an earlier return of cyclic variation of integrated backscatter than of wall thickness in stunned myocardium, so the relationship between wall thickness and integrated backscatter is maintained.

Acknowledgements - These investigations were supported by grant RGN02332 from the Netherlands Technology Foundation (STW).

\section{REFERENCES}

Barzilai B, Madaras EI, Sobel BE, Miller JG, Perez JE. Effects of myocardial contraction on ultrasonic backscatter before and after ischemia. Am J Physiol 1984;247:H478-H483.

Bloomfield P. Fourier analysis of time series: An introduction. New York: John Wiley, 1976:80-85.

Bolli R, Zhu WX, Thomby JI, O'Neill PG, Roberts R. Time course and determinants of recovery of function after reversible ischemia in conscious dogs. Am J Physiol 1988;254:H102-H114.

Brand T, Sharma HS, Fleischmann KE, et al. Proto-oncogene expression in porcine myocardium subjected to ischemia and reperfusion. Circ Res 1992;71:1351-1360.

Glueck RM, Mottley JG, Sobel BE, Miller JG, Pérez JE. Changes in ultrasonic attenuation and backscatter of muscle with state of contraction. Ultrasound Med Biol 1985;11:605-610.

Haasler GB, Rhyne TL, Komorowski R, Boerboom LE, Sagar KB The lack of effect of hemodilution, myocardial water content, and increased coronary artery blood flow on integrated backscatter in the beating canine heart. Ultrason Imaging 1993;15:25-35.

Hearse DJ. Stunning: Three questions and concerns. Basic Res Cardiol 1995;90:263-265.

Hoffmeister BK, Wong AK, Verdonck ED, Wickline SA, Miller JG. Anisotropy of ultrasonic backscatter from human tendon compared to that from normal human myocardium. IEEE Trans UFFC 1991;91CH3079-1:1127-1131.

Lancée CT, Mastik F, Rijsterborgh H, Bom N. Myocardial backscatter analysis in animal experiments. Ultrasonics 1988;26:155163.

Maderas EI, Barzilai B, Pérez JE, Sobel BE. Miller JG. Changes in myocardial backscatter throughout the cardiac cycle. Ultrason Imaging 1983;5:229-239.

Maderas EI, Pérez J, Sobel BE, Mottley JG, Miller JG. Anisotropy of the ultrasonic backscatter of myocardial tissue: II. Measurements in vitro. J Acoust Soc Am 1988;83:762-769.

McPherson DD, Skorton DJ, Wilbricht CJ, et al. Is myocardial perfusion a determinant of integrated ultrasound backscatter. Clin Res 1986; 34:898A

Miller JG, Pérez JE, Mottley JG, et al. Myocardial tissue characterization: An approach based on quantitative backscatter and attenuation. Proc IEEE Ultrasonics Symp 1983; CH1947-1:782-793.

Milunski MR, Mohr GA, Wear KA, et al. Early identification with ultrasonic integrated backscatter of viable but stunned myocardium in dogs. J Am Coll Cardiol 1989a; 14:462-471.

Milunski MR, Mohr GA, Pérez JE, et al. Ultrasonic tissue characterization with integrated backscatter: Acute myocardial ischemia, reperfusion, and stunned myocardium in patients. Circulation $1989 b ; 80: 491-503$.

Mimbs WJ. Bauwens D, Cohen RD. et al. Effects of myocardial ischemia on quantitative ultrasonic backscatter and identification of responsible determinants. Circ Res 1981:49:89-96.

Mohr GA. Vered Z, Barzilai B, et al. Automated determination of the magnitude and time delay ("phase") of the cardiac cycle dependent variation of myocardial ultrasonic integrated backscatter. Ultrason Imaging 1989; 11:245-259.

Mottley JG, Glueck RM, Pérez JE, Sobel BE, Miller JG. Regional 
differences in the cyclic variation of myocardial backscatter that parallel regional differences in contractile performance. J Acoust Soc Am 1984; 76:1617-1623.

Mottley JG, Miller JG. Anisotropy of the ultrasonic backscatter of myocardial tissue: I. Theory and measurements in vitro. J Acoust Soc Am 1988;83:755-761.

O'Brien WD, Sagar KB, Warltier DC, Rhyne TL. Acoustic propagation of normal, stunned and infarcted myocardium: Morphological and biochemical determinants. Circulation 1995;91:154-160.

Office of Science and Health Reports. Guide for the care and use of laboratory animals. DHEW Publication No. (NIH) 80-23, DRR/ NIH Bethesda.

Recchia D, Miller JG, Wickline SA. Quantification of ultrasonic anisotropy in normal myocardium with lateral gain compensation of two dimensional integrated backscatter images. Ultrasound Med Biol 1993; 19:497-505.

Recchia D, Hall CS, Shepard RK, Miller JG, Wickline SA. Mechanisms of the view dependence of ultrasonic backscatter from normal myocardium. IEEE Trans UFFC 1995;42:91-98.

Rijsterborgh H, Mastik F, Lancée CT, et al. Ultrasonic myocardial integrated backscatter and myocardial wall thickness in animal experiments. Ultrasound Med Biol 1990;16:29-36.

Rijsterborgh H, Mastik F, Lancée CT, et al. The relative contributions of myocardial wall thickness and ischemia to ultrasonic myocardial integrated backscatter during experimental ischemia. Ultrasound Med Biol 1991;17:41-48.

Rijsterborgh H, Mastik F, Lancée CT, et al. Ultrasound myocardial integrated backscatter signal processing: Frequency domain versus time domain. Ultrasound Med Biol 1993;19:211-219.

Rijsterborgh H, van der Steen AFW, Mastik F, et al. The relationship between myocardial integrated backscatter and wall thickness during isovolumic contraction: An isolated pig heart study. Ultrasound Med Biol 1996;22:43-52.

Sagar KB, Rhyne TL, Warltier DC, Pelc LE, Wann LS. Intramyocardial variability in integrated backscatter: Effects of coronary occlusion and reperfusion. Circulation 1987;75:436-442.

Sagar KB, Pelc LE, Rhyne TL, Wann LS, Warltier DC. Influence

Volume 23, Number 3, 1997

of heart rate, preload, afterload, and inotropic state on myocardial ultrasonic backscatter. Circulation 1988; 77:478-483.

Sagar KB, Pelc LR, Rhyne TL, et al. Role of ultrasonic tissue characterization to distinguish reversible from irreversible myocardial injury. J Am Soc Echocardiogr 1990;3:471-477.

Snei I.K, Sassen LMA, Fan DS, et al. Mynfibrillar $\mathrm{Ca}^{2+}$ sensitization predominantly enhances function and mechanical efficiency of stunned myocardium. Circulation 1994;90:959-969.

van der Steen AFW, Rijsterborgh H. Myocardial ultrasonic tissue characterization: The Rotterdam approach. Thorax Centre J 1996;8:25-29.

van der Steen AFW, Rijsterborgh H, Mastik F, et al. Influence of attenuation on measurements of ultrasonic myocardial integrated backscatter during the cardiac cycle. Ultrasound Med Biol $1991 ; 17: 869-877$.

van der Steen AFW, Rijsterborgh H, Krams R, et al. Cyclic variation of integrated backscatter and wall thickness in stunned myocardium. Ultrasonogr Bull 1995;23:6-10.

Wear KA, Shoup TA, Popp RL. Ultrasonic characterization of canine myocardium contraction. IEEE Trans UFFC 1986;33:347353.

Wear KA, Milunski MR, Wickline SA, et al. Contraction-related variation dependence of acoustic properties of canine myocardium. J Acoust Soc Am 1989;86:2067-2072.

Wickline SA, Thomas LI III, Miller JG, Sobel BE, Pérez. IE. The dependence of myocardial ultrasonic integrated backscatter on contractile performance. Circulation 1985a; 72:183-192.

Wickline SA, Thomas LJ III, Miller JG, Sobel BE, Pérez JE. A relationship between ultrasonic integrated backscatter and myocardial contractile function. J Clin Invest 1985b; 76:2151-2160.

Wickline SA, Verdonk ED, Miller JG. Three-dimensional characterization of human ventricular myofiber architecture by ultrasonic backscatter. J Clin Invest 1991;88:438-446.

Wickline SA, Verdonk ED, Wong AK, Shepard RK, Miller JG Structural remodeling of human myocardial tissue after infarction: Quantification with ultrasonic backscatter. Circulation 1992;85:259-268. 